\title{
MODEL-BASED INSULIN SENSITIVITY FOR EARLY DIAGNOSIS OF SEPSIS IN CRITICAL CARE
}

Wan Fadzlina Wan Muhd Shukeri ${ }^{1}$, Azrina Md. Ralib ${ }^{1}$, Ummu Khultum Jamaludin ${ }^{2}$ and Mohd Basri Mat-Nor ${ }^{1}$

${ }^{1}$ Department of Anesthesiology and Critical Care, Kuliyyah of Medicine, International Islamic University of Malaysia, ${ }^{2}$ Faculty of Mechanical Engineering, Universiti Malaysia Pahang, Malaysia.

Presenter: Wan Fadzlina Wan Muhd Shukeri, wfwms82@yahoo.co.uk

Introduction: Currently, it is almost impossible to diagnose a patient at the onset of sepsis due to the lack of real-time metrics with high sensitivity and specificity. The purpose of the present study is to determine the diagnostic value of model-based insulin sensitivity $(\mathrm{SI})$ as a new sepsis biomarker in critically ill patients, and compare its performance to classical inflammatory parameters.

Materials and method: We monitored hourly SI levels in septic $(n=19)$ and non-septic $(n=19)$ critically ill patients in a 24-hour follow-up study. Patients with type I or type II diabetes mellitus were excluded. SI levels were calculated by a validated glycemic control software, STAR TGC (Stochastic TARgeted Tight Glycemic Controller) (Christchurch, NZ). STAR TGC uses a physiological glucose-insulin system model coupled with stochastic models that capture SI variability in real time.

Results: The median SI levels were lower in the sepsis group than in the non-sepsis group $(1.9 \times 10-4 \mathrm{~L} / \mathrm{mU} / \mathrm{min}$ vs $3.7 \times 10-4 \mathrm{~L} / \mathrm{mU} / \mathrm{min}, \mathrm{P}$ \& $1 \mathrm{t} ; 0.0001)$. The areas under the receiver operating characteristic curve (AUROC) of the model-based SI for distinguishing non-sepsis from sepsis was 0.911, superior to white cells count (AUROC 0.611) and temperature (AUROC 0.618). The optimal cut-off value of the test was $2.9 \times 10-4$ $\mathrm{L} / \mathrm{mU} / \mathrm{min}$. At this cut-off value, the sensitivity and specificity was $88.9 \%$ and $84.2 \%$, respectively. The positive predictive value was $84.2 \%$, while the negative predictive value was $88.9 \%$.

Conclusion: The early and relevant decrease of SI in sepsis suggests that it might be a promising novel biomarker of sepsis in critical care. Low SI is diagnostic of sepsis, while high SI rules out sepsis, and these may be determined non-invasively in real-time from glycemic control protocol data. 